\section{The surgical management of primary hyperparathyroidism: an updated review}

\author{
Kaela E Parnell ${ }^{1}$ \& Sarah C Oltmann*,1 \\ ${ }^{1}$ Department of Surgery, University of Texas Southwestern Medical Center, 5323 Harry Hines Boulevard, E6.104B, Dallas, TX \\ 75390-9092, USA \\ *Author for correspondence: Tel.: +1 214648 6439; Fax: +1 214648 9448; sarah.oltmann@utsouthwestern.edu
}

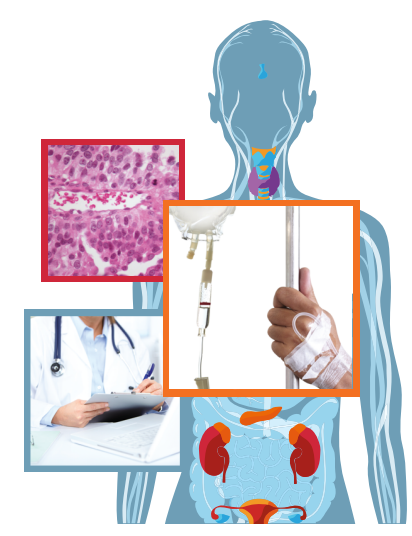

International Journal of Endocrine Oncology

Patients with primary hyperparathyroidism often present clinically asymptomatic with various biochemical compositions of serum calcium, parathyroid hormone, vitamin D and urinary calcium. Understanding the subtle differences in clinical and biochemical presentations is key for timely diagnosis and referral to an experienced parathyroid surgeon. Surgery remains the only option for cure of primary hyperparathyroidism, which now favors a directed parathyroidectomy with intra-operative adjuncts. However it is important to understand and revise the surgical approach for patients with hereditary conditions or nonlocalizing studies. Revised guidelines from the Fourth International Workshop on the Management of Asymptomatic Primary Hyperparathyroidism in 2013 and the American Association of Endocrine Surgeons in 2016 are reviewed in this paper for an updated review of this condition.

First draft submitted: 5 July 2017; Accepted for publication: 19 March 2018; Published online: 23 May 2018

Keywords: asymptomatic primary hyperparathyroidism $\bullet$ hereditary hyperparathyroidism $\bullet$ intraoperative adjuncts $\bullet$ intraoperative parathyroid hormone $\bullet$ parathyroid adenoma $\bullet$ parathyroid hyperplasia $\bullet$ primary hyperparathyroidism - surgical indications parathyroidectomy

Primary hyperparathyroidism (PHPT) is an intrinsic abnormality of one or more parathyroid glands in which the abnormal parathyroid gland inappropriately secretes parathyroid hormone (PTH) relative to the serum calcium. Autonomous secretion of PTH in patients with PHPT is attributed to one of three etiologies: single or multiple adenomas, multigland hyperplasia or carcinoma. Approximately $80-85 \%$ of cases are due to parathyroid adenomas, $10-15 \%$ are due to multigland hyperplasia, and less than $1 \%$ are attributed to parathyroid carcinomas [1]. A myriad of deleterious effects can occur as a result of elevated serum PTH and calcium including osteoporosis and bone disease, nephrolithiasis, pancreatitis, peptic ulcer disease, along with several neuropsychiatric and gastrointestinal symptoms. Surgery remains the only option for cure for patients with PHPT. Given that the vast majority of cases are due to a single adenoma, the surgical approach and management has significantly changed over the last two decades from a standardized bilateral neck exploration to a directed approach focused on excising the pathologic gland. This approach is made possible with the advancement of preoperative localization studies and intraoperative adjuncts and it has become an accepted and effective technique when performed by an experienced surgeon [2]. Curative results exceed $98 \%$ with nerve injury rates usually less than $1 \%[3,4]$. Updated recommendations were developed at The Fourth International Workshop on the Management of Asymptomatic Primary Hyperparathyroidism which took place in Florence, Italy in September of 2013 [5]. The American Association of Endocrine Surgeons also provided new evidence-based guidelines in August of 2016 [6]. The purpose of this paper is to review the current diagnosis and surgical management of PHPT within the context of the most recent guidelines.

\title{
Diagnosis
}

PHPT is a biochemical diagnosis consisting of an elevated serum calcium and PTH, or an inappropriately normal (i.e., nonsupressed) serum PTH in the setting of normal renal function. If the urinary calcium is also elevated, the diagnosis of PHPT is confirmed. Decreased serum phosphate may also be noted in some patients. The biochemical workup for a patient who is suspected of having PHPT should include measuring serum calcium, PTH, creatinine, 25-hydroxyvitamin D (25[OH]-vitamin D), along with 24-h urinary calcium and creatinine (Box 1).

Future Medicine 
Box 1. Biochemical panel to obtain when considering primary hyperparathyroidism.

Recommended biochemical evaluation for PHPT:

- Total calcium (ionized calcium when applicable)

- PTH

- Creatinine

- 25-hydroxyvitamin D

- 24-h urinary calcium and creatinine

Obtain ionized calcium when serum total calcium is within normal ranges in the setting of elevated PTH. This will differentiate between the normocalcemic variant of PHPT and the classic hypercalcemic PHPT presentation.

PHPT: Primary hyperparathyroidism; PTH: Parathyroid hormone.

Box 2. Biochemical variants of primary hyperparathyroidism.

PHPT: three common presentations

- Elevated calcium and PTH (classic form)

- Elevated calcium and normal PTH (nonsuppressed PTH)

- Normal calcium and elevated PTH (normocalcemic PHPT) ${ }^{\dagger}$

${ }^{\dagger}$ If considering normocalcemic PHPT, one must rule out causes of secondary hyperparathyroidism such as chronic kidney disease, vitamin D deficiency and gastrointestinal malabsorption problems such as short gut syndrome, Crohn's disease or a prior Roux-en-Y bypass surgery.

PHPT: Primary hyperparathyroidism; PTH: Parathyroid hormone.

\section{Document hypercalcemia}

Total serum calcium levels in $\mathrm{mg} / \mathrm{dl}$ or $\mathrm{mmol} / \mathrm{l}$ should be measured in the patient being considered for PHPT. Physicians should have a low threshold to also evaluate calcium via an ionized level. Repeated measurements are required because patients can be normocalcemic intermittently, although hypercalcemic a majority of the time [7]. There are three biochemical forms of PHPT and it is important to recognize the discrepancies between each (Box 2). The classic presentation is the hypercalcemic form of PHPT in which patients demonstrate elevated serum calcium and PTH concentrations. The second variant is observed when patients demonstrate hypercalcemia however PTH levels are within normal reference ranges (i.e., inappropriately nonsuppressed PTH). The third presentation is 'normocalcemic PHPT' in which PTH levels are elevated with a normal total and ionized serum calcium concentration. Thus when PTH is elevated but the serum total calcium is normal, an ionized serum calcium is required to confirm the diagnosis. If the ionized calcium is elevated, the patient has traditional HPT and is not considered eucalcemic or normocalcemic. Obtaining an ionized serum calcium is unnecessary when the initial total calcium level is elevated, as in the classic form of PHPT. For patients with suspected normocalcemic PHPT, disorders causing secondary hyperparathyroidism must be excluded such as chronic kidney disease, vitamin $\mathrm{D}$ deficiency and gastrointestinal malabsorption problems such as short gut syndrome, Crohn's disease or a prior Roux-en-Y bypass surgery $[6,7]$. For those with chronic kidney disease, population studies demonstrate that PTH begins to rise when the estimated glomerular filtration rate declines to $60 \mathrm{ml} / \mathrm{min} / 1.73 \mathrm{~m}^{2}$ or less [7]. When PHPT patients are vitamin D-deficient, they may initially present normocalcemic but can become hypercalcemic after vitamin D supplementation and shift to the classic form of PHPT [6]. However, since vitamin D deficiency is also a cause of elevated PTH, when vitamin D repletion takes place in a patient without PHPT and with normal calcium levels, the PTH level may return to normal without the development of hypercalcemia. There is also evidence suggesting that normocalcemic PHPT may be the first phase in a biphasic disorder progressing to hypercalcemic PHPT $[8,9]$. While the most common cause of hypercalcemia in adults is PHPT and malignancy, other etiologies of hypercalcemia should be explored with a full review of medications, including thiazide diuretics, calcium supplementation and the history of or present lithium use [7].

\section{Document elevated PTH}

Eastell et al. recommends three documented instances of elevated PTH over a 3-6-month period [7], keeping in mind that a 'nonsuppressed' PTH within normal reference levels is physiologically not normal in the setting of hypercalcemia and consistent with PHPT. As mentioned earlier, disorders causing compensatory or secondary 
hyperparathyroidism must be excluded, such as vitamin D deficiency and chronic kidney disease. Patients with PHPT who are also vitamin-D-deficient $(<20 \mathrm{ng} / \mathrm{ml})$ as defined by the Institute of Medicine, tend to produce higher serum levels of PTH [10]. In the setting of PHPT, vitamin D repletion has been associated with reductions in the PTH level with little effect on serum calcium concentrations [11-13] and varying effects on urinary calcium concentrations [12,13]. Some experts believe vitamin D values must be above $30 \mathrm{ng} / \mathrm{ml}$ as recommended by the Endocrine Society when evaluating concomitant PTH levels because PTH concentrations may continue to be secondarily elevated when $25(\mathrm{OH})$-vitamin D levels are below this value $[14,15]$. Established reference guidelines for PTH values based on co-existing $25(\mathrm{OH})$-vitamin D concentrations do not currently exist [7,16]. A lower reference PTH range would be obtained if a higher threshold for vitamin D repletion was used [7]. Vitamin D concentrations are also affected by factors such as gender, BMI, age, race and menopausal status, which should also be considered when evaluating PTH levels. Various institutions have proposed a multidimensional PTH calculator or nomogram incorporating several influential factors $[14,17,18]$, however these tools have not been incorporated into formalized guidelines.

\section{Document vitamin D level}

It is recommended that $25(\mathrm{OH})$-vitamin D levels be measured in all patients with PHPT. No additional information is gained by measuring 1,25-dihydroxyvitamin $\mathrm{D}$ and it is unnecessary [7]. Recommended vitamin $\mathrm{D}$ levels are controversial and vary with race. The Institute of Medicine recommends vitamin D threshold levels above $20 \mathrm{ng} / \mathrm{ml}$ ( $50 \mathrm{nmol} / \mathrm{l})$, which is supported by Eastell et al. [7] while the Endocrine Society recommends a threshold of $30 \mathrm{ng} / \mathrm{ml}(75 \mathrm{nmol} / \mathrm{l})$. As mentioned previously, the diagnostic accuracy (sensitivity and specificity) for PHPT is increased when vitamin D is replete [19]. The American Association of Endocrine Surgeons recommends vitamin D supplementation to operative candidates who are deficient prior to performing a parathyroidectomy, however this is a 'weak recommendation' with 'low quality evidence', and repletion should be performed carefully [6]. It is common practice however for many surgeons to wait until the postoperative period to provide vitamin $\mathrm{D}$ supplementation due to the risk of exacerbating hypercalcemia and hypercalciuria. It may take 6-12 months to see reductions in PTH even after vitamin D is replete [7]. After parathyroidectomy, serum 25(OH)-vitamin D levels usually increase without additional supplementation [20].

\section{Exclude familial hypocalciuric hypercalcemia}

It is important to measure 24-h urinary calcium and creatinine levels when evaluating a patient for PHPT in order to rule out familial hypocalciuric hypercalcemia $(\mathrm{FHH})$. FHH is an autosomal dominant condition in which the CaSR gene encoding the calcium sensing receptor has a heterozygous inactivating mutation, which causes an abnormal parathyroid and renal calcium set point [21]. Patients usually have elevated serum calcium and PTH levels with low urinary excretion of calcium. The diagnosis is made when the urinary calcium is less than $100 \mathrm{mg} / 24 \mathrm{~h}$ and the calcium to creatinine clearance ratio is less than 0.01 [5,22]. Patients must be off thiazide diuretics during this test. Patients with FHH are usually asymptomatic, have a long standing personal and family history of hypercalcemia, and do not benefit from surgery. If suspected, genetic testing is recommended for CaSR, GNA11, and AP2S1 genes which evaluates for FHH1, FHH2, and FHH3, respectively [23] and patients should be managed medically.

\section{Clinical presentation}

Just as the biochemical presentation varies in patients with PHPT, the clinical presentation is similarly heterozygous. Patients may have overt signs and symptoms of PHPT including: kidney stones, osteoporosis, pathological fractures, pancreatitis, gastric ulcers, and hypercalcemic crises. However in the USA and Western Europe, asymptomatic PHPT has been the most common clinical phenotype for the past 40 years [24]. Although overt symptoms are usually not present, there is typically evidence of end-organ damage such as nephrolithiasis, reductions in bone mineral density (BMD), or decreased renal function. Patients may also present with a milieu of nonspecific symptoms, including subtle neuropsychiatric, cognitive, musculoskeletal, or gastrointestinal complaints. Data regarding the natural course of asymptomatic PHPT are limited [24]. Prospective studies show that in 15 years, a third of subjects demonstrate overt features of PHPT such as worsening hypercalcemia, kidney stones and reduced BMD [10]. Despite having clear evidence showing reductions in BMD in patients with PHPT, data are limited on the fracture risk in patients with PHPT based upon dual-energy x-ray absorptiometry (DEXA) or other quantitative modalities [24]. It is unclear whether $\mathrm{T}$ scores or fracture risk assessment scores (FRAX) would predict fractures in patients with PHPT in the same way it does in patients without PHPT [24]. 
There also appears to be geographical differences in the phenotypes of PHPT. Epidemiological studies from Latin American and Chinese regions show an evolution toward the asymptomatic disease possibly due to increasing screening tests that include serum calcium along with incidental findings of parathyroid nodules on thyroid ultrasonography [24]. Other Asian countries, including India, Iran, Saudi Arabia and Thailand still report predominantly symptomatic disease with renal and skeletal manifestations, possibly due to less access to preventative screening evaluations [24].

PHPT can also be manifested as part of a genetic condition. Hereditary forms of PHPT account for $5-10 \%$ of cases [2,25-26]. The likelihood of a genetic condition increases with younger patients, less than 40 years of age with multigland disease, who may have a family history of nephrolithiasis, premature osteoporosis or other signs of hypercalcemia along with various tumors [6]. Genetic screening is indicated in high risk patients and should include testing for the following conditions: MEN 1 syndrome with MEN1 gene mutations, in which patients may present with peptic ulcers and other endocrinopathies such as pituitary, pancreaticoduodenal or thymic tumors along with occasional bronchial carcinoids [2]; MEN 2A syndrome with RET oncogene mutations, in which medullary thyroid cancer is also prevalent; hyperparathyroidism-jaw tumor (HPT-JT) syndrome with HRPT2/CDC73 parafibromin gene mutations, in which patients have a higher incidence of parathyroid carcinoma along with renal cysts, adult Wilm's tumor, uterine carcinoma and tumors of the mandible [2]. Genetic testing not only confirms the diagnosis, but it allows for appropriate screening and treatment for other disease processes which affects the timing and extent of parathyroidectomy [6].

\section{Parathyroid tumorigenesis}

Parathyroid tumorigenesis is attributed to abnormalities in key growth-controlling genes, tumor suppressor genes, or proto-oncogenes, which usually cause monoclonal cell proliferation in both solitary adenomas and carcinomas [27]. As mentioned above, loss-of-function mutations take place in tumor suppressor genes such as MEN 1 in MEN 1 syndrome and HRPT2/CDC73 in HPT-JT syndrome, triggering cellular proliferation [2]. Sporadic (nonfamilial) parathyroid carcinomas are also linked to mutations of the HRPT2/CDC73 tumor suppressor gene [28] along with cyclin D1 and PI3K/AKT/MTOR pathway genes [29]. Additional unidentified genes have been implicated in parathyroid cancer with ongoing research currently taking place. Conversely, the RET proto-oncogene undergoes a gain-of-function mutation responsible for the development of MEN 2A syndrome [2]. Similarly, overexpression of cyclin D1 results in the proliferation seen in sporadic tumors, which is caused by the relocation of PRAD1 oncogene on chromosome 11 [27]. This ultimately leads to excessive parathyroid cell proliferation and dysregulation of the secretory control of PTH by calcium. Somatic and germline mutations of $C D K N 1 B$, encoding p27 cyclin-dependent kinase inhibitor (CDKI), have also been linked to the development of sporadic parathyroid adenomas [30].

\section{Surgical indications}

Once the diagnosis of PHPT has been confirmed, determining management is the next step. Surgery is the only option for cure in patients with PHPT. There is a universal agreement that the following criteria are indications for surgery in patients with PHPT [6,22] (Box 3):

- All patients $<50$ years of age, regardless of whether objective features are present or absent [6];

- All symptomatic patients, including those with kidney stones, hypercalcemic crises, pathologic fractures or other associated symptoms;

- Patients with findings concerning for parathyroid cancer;

- All asymptomatic patients with the following:

- Serum calcium $>1.0 \mathrm{mg} / \mathrm{dl}(0.25 \mathrm{mmol} / \mathrm{l})$ above the normal range;

- BMD by DEXA: T-score $\leq 2.5$ at the lumbar spine, total hip femoral neck or distal $1 / 3$ radius $[6,10]$;

- Vertebral fracture by x-ray, computed tomography (CT), MRI and vertebral fracture assessment;

- Estimated glomerular filtration rate of less than $60 \mathrm{ml} / \mathrm{min} \mathrm{[6];}$

- Urinary calcium excretion $>400 \mathrm{mg}$ in $24 \mathrm{~h}$;

- Nephrolithiasis or nephrocalcinosis by $\mathrm{x}$-ray, ultrasound or CT.

- Asymptomatic patients who cannot participate in appropriate medical surveillance;

- Asymptomatic patients desiring definitive surgical management. 
Box 3. Indications for parathyroidectomy in primary hyperparathyroidism.

- Indications for parathyroidectomy in the setting of primary hyperparathyroidism

- Age $<50$

- All symptomatic patients

- Signs/symptoms concerning for malignancy

- Asymptomatic patients with the following:

- Serum Ca $>1.0 \mathrm{mg} / \mathrm{dl}$ above normal range

- Dual-energy x-ray absorptiometry T-score $\leq 2.5$ at lumbar spine, femoral neck or distal radius

- Vertebral fractures

- Estimated glomerular filtration rate $<60 \mathrm{ml} / \mathrm{min} / 1.73 \mathrm{~m}^{2}$

- Urinary Ca excretion $>400 \mathrm{mg} / 24 \mathrm{~h}$

- Nephrolithiasis and nephrocalcinosis

- Asymptomatic patients unable to undergo medical surveillance

- Asymptomatic patients desiring definitive surgical management

- All patients with PHPT should be referred to an endocrine surgeon. This box summarizes the indications for surgery.

There is significant evidence to support surgery as the initial treatment option for patients with symptomatic and asymptomatic PHPT. Cure rates in sporadic PHPT approach 95-99\% after parathyroidectomy, which is defined as the establishment of normocalcemia lasting a minimum of 6 months [6]. After parathyroidectomy, patients are shown to have reductions in bone fracture incidence [10], normalization of bone turnover markers and increases in BMD at all sites by DEXA imaging, which is greatest and most rapid at the lumbar spine and hip, followed by the distal radius [10,24]. Although nephrocalcinosis and renal insufficiency do not resolve, there is a decrease in the development of new kidney stones after surgery, which may also slow the decline of glomerular filtration rate [6,31]. Several nonspecific symptoms such as gastroesophageal reflux disease, fatigue, bone and joint pain, muscle aches, nocturia, polydipsia, headaches, nausea, vomiting, difficulty concentrating, irritability, memory problems, anxiety, sleep problems, depression, abdominal pain, constipation, and urinary incontinence have also been shown to improve after parathyroidectomy $[6,32,33]$. There is evidence showing that patients with PHPT have higher rates of hypertension, myocardial infarction, congestive heart failure, stroke, diabetes and overall mortality [6]. Studies show an improvement in several cardiac parameters including hypertension, left ventricle hypertrophy and diastolic dysfunction in those who undergo parathyroidectomy [34-38], however some conflicting data exist [6]. Improvement of neuropsychiatric and neurocognitive function after parathyroidectomy has been reported, including increased focus, mood and quality of life [6,10,24]. However these data are inconsistent with insufficient evidence at the current time to formally recommend surgery on the basis of cardiac or neuropsychiatric improvement [10,24].

For those patients with significant medical comorbidities and a limited life expectancy, parathyroidectomy should be approached with caution. While parathyroidectomy can generally be done safely, and various anesthesia strategies are feasible, it is critical to weigh the risks and benefits of the procedure [39]. If the patient is struggling with symptomatic hypercalcemia, parathyroidectomy should be entertained, whereas an asymptomatic patient with significant medical comorbidities and a limited life expectancy may not obtain much benefit from the intervention.

There is clear evidence showing progression to symptomatic PHPT in up to a third of patients who do not undergo surgery [10]. Therefore, asymptomatic patients who do not desire surgery should be followed closely by an endocrinologist or primary-care physician along with mineral metabolism clinic follow-up to ensure annual evaluations of serum calcium, PTH, vitamin D, creatinine, glomerular filtration rate, 24 h urinary tests and periodic DEXA scans every 1-2 years [6,10]. Calcium intake should follow guidelines for all individuals established by the Institute of Medicine and should not be limited in patients with PHPT who do not qualify for surgery [6,10,40]. Vitamin D repletion should be performed cautiously because there is a risk of exacerbating hypercalcemia and hypercalciuria. Recommended regimens aim to increase 25(OH)-vitamin D concentrations to 20-30 ng/ml (50$75 \mathrm{nmol} / \mathrm{l}$ ) [10,40]. Pharmacologic agents such as calcimimetics and bisphosphonates have been used to decrease serum calcium and improve BMD, respectively. Calcimimetics reduce calcium levels but do not improve BMD [40] and only show modest reductions in PTH [40]. Alendronate has been shown to increase BMD at the lumbar spine and hip, while decreasing bone turnover markers without affecting calcium or PTH levels in patients with PHPT [40]. There is minimal data on fracture rates associated with bisphosphonate therapy in PHPT [40]. Combination therapy using calcimimetics and bisphosphonates appears to achieve both goals of lowering serum levels of calcium and 
stabilizing BMD [40]. However surgical management is more successful and cost effective than pharmacologic therapy [6] and it remains the treatment of choice, as it is the only means for cure.

\section{Preoperative evaluation}

There are important aspects of the preoperative evaluation specifically pertinent to patients with PHPT. A history of prior neck irradiation, whether used to treat breast cancer [41], lymphoma, head and neck malignancies or benign conditions such as acne has been associated with increased risk for parathyroid disease [42,43]. Prior neck surgery or radiation will cause more cervical scarring, making the operation more difficult. Evaluation of vocal cord function is mandatory in these patients as well as in those already with signs of vocal cord dysfunction. Indirect mirror examination, flexible fiber optic laryngoscopy or recently developed techniques such as laryngeal ultrasonography [44,45] are all acceptable methods for evaluation. All medications should be reviewed, especially those that affect calcium or PTH including lithium, anticonvulsants, calcium-containing products, anticoagulants, thiazide diuretics and vitamin D. Lithium-associated PHPT occurs in up to $15 \%$ of long-term users $[6,46]$. It is important that thyroid goiters and nodular disease be assessed preoperatively, with potential biopsies when indicated for the purpose of addressing both problems at the initial operation. When reviewing DEXA imaging, decreases in BMD are usually most pronounced at cortical bone sites such as the distal radius and it is therefore imperative to include this site during the evaluation [6]. Newer imaging modalities may be more sensitive than DEXA in detecting decreases in trabecular BMDs, however recommendations based on these modalities have not been validated.

When patients present in hypercalcemic crisis, the surgeon must facilitate stabilizing the patient prior to proceeding to expeditious parathyroidectomy. Hypercalcemic crisis typically presents rapidly, with a serum calcium level greater than $14 \mathrm{mg} / \mathrm{dl}$ and signs of multi-organ dysfunction [47]. The goal of initial treatment in these instances focuses on bringing down serum calcium levels, ideally into a eucalcemic state. This requires fluid resuscitation with normal saline, management with bisphosphonates to shift serum calcium toward the bones and furosemide to excrete calcium into the urine.

\section{Preoperative imaging}

Imaging is an important localizing method that should be ideally ordered by the surgeon due to the high variability between different studies and institutions. It is not a diagnostic study and has no utility in excluding or confirming the diagnosis of PHPT [6]. There is no reason to obtain an imaging study if surgery is not planned and negative or nonlocalizing imaging should not inhibit referral to an experienced parathyroid surgeon $[2,48]$. These results would simply necessitate a four-gland exploration as opposed to a directed parathyroidectomy. The surgeon should work in concert with the nuclear medicine physician and radiologist to evaluate the quality of results for optimal operative planning. Imaging options include: cervical ultrasound, nuclear scintigraphy, a sestamibi scan, for example, 4-dimension computed tomography (4D CT), MRI and venous sampling. The most cost effective strategy is cervical ultrasound by an experienced sonographer, combined with sestamibi imaging or 4D CT [6,49-51]. Cervical ultrasound is advantageous due to its low expense, lack of ionizing radiation and ability to evaluate the thyroid for concomitant pathology. It can be performed by the operating surgeon and repeated in the operating room on the day of surgery. Limitations include difficulty in evaluating retroesophageal or mediastinal lesions and it is highly operator dependent. Localization accuracy and sensitivity increases when ultrasonography is combined with sestamibi imaging [6]. Technetium (Tc 99m) sestamibi is the most common radioisotope in parathyroid scintigraphy and can be combined with single-photon emission computed tomography which can identify ectopic parathyroid glands, including mediastinal or retroesophageal glands. The radiation dose is minimal and it is relatively inexpensive, however the sensitivity in multigland disease is poor [6]. Although traditional CT has little utility, 4D CT protocol has increased sensitivity with excellent anatomic detail. 4D CT, however, requires a high level of radiologic expertise, lacks sensitivity in multigland disease [6], and causes significantly higher radiation exposure than sestamibi. It is unlikely for any imaging study to demonstrate enlargement of all four parathyroid glands, instead four-gland hyperplasia should be considered when there is enlargement of more than one gland [40]. Venous sampling and MRI can be considered in cases of re-operation, contraindications to radiation or difficult localization [6]. Preoperative fine-needle aspiration (FNA) of parathyroid lesions is not recommended and rarely necessary [6]. FNA biopsy can cause trabecular scarring that mimics the appearance of cancer, which would necessitate a more extensive resection and confound the surgical decisions. 


\section{PHPT \& concomitant thyroid disease}

Patients with PHPT are found to have concomitant thyroid nodules with frequencies ranging from 20 to $60 \%$, while patients presenting with thyroid disease are found to have PHPT at a much lower rate, ranging from 0.3 to $1 \%$ in most studies [52]. Since the surgical management of PHPT has evolved to a focused, unilateral approach, thyroid nodules and cancer can be missed during the index operation. For this reason, preoperative ultrasonography should be performed on all PHPT patients for the purposes of localizing parathyroid adenomas as well as detecting concomitant thyroid nodules. If thyroid nodules meet criteria, FNA should be performed followed by the appropriate thyroid resection during the same initial parathyroidectomy operation. Alternatively, it is recommended that serum PTH and calcium concentrations be evaluated preoperatively in patients presenting with thyroid disease to also address both diseases simultaneously [52].

\section{Surgical approaches $\&$ intraoperative adjuncts}

Two main surgical approaches have evolved among parathyroid surgeons involving the use of four-gland exploration versus directed parathyroidectomy. Both techniques have yielded excellent results with minimal complication rates when performed by an experienced parathyroid surgeon [2,53,54]. The surgical volume that defines an experienced surgeon is variable among the literature but ranges between 20 and 50 parathyroidectomies annually, which has shown to result in lower costs, lower complication rates and decreased rates of persistent or recurrent PHPT [55-57].

Bilateral cervical exploration under general anesthesia has historically been the standard of care for definitive treatment of PHPT, regardless of preoperative imaging. This is still preferred at some institutions due to the inability of preoperative imaging to consistently identify local disease [2]. It mandates that all four parathyroid glands be identified and assessed, to determine whether multigland disease versus a single adenoma exists. Success rates exceed $95 \%$ when performed by an experienced parathyroid surgeon with low complication rates of less than $4 \%[58,59]$. Four-gland exploration can be performed through a small cosmetically appearing neck incision in the outpatient setting. This approach is preferred in cases with multigland disease, nonlocalizing preoperative imaging, hereditary forms of hyperparathyroidism, lithium-induced disease or those who fail to meet the 'Miami criterion' [60] of intraoperative PTH monitoring during a directed parathyroidectomy. Multigland hyperplasia can occur in $10-15 \%$ of patients with sporadic PHPT, influencing some experts to advocate for bilateral exploration despite intraoperative PTH levels decreasing appropriately to satisfy the Miami criterion [2,61]. This is due to the primary concern that residual enlarged parathyroid glands remaining in situ can cause recurrent or persistent disease $[2,61]$.

In comparison, directed parathyroidectomy is a focused, imaged-guided technique that targets the presumed hyperfunctioning parathyroid adenoma without necessarily identifying additional parathyroid glands. Intraoperative adjuncts are used to determine whether further pathologic gland(s) remain in situ. The basis of this approach is that $85 \%$ of PHPT is due to a single parathyroid adenoma, and when excised, yields a reliable and durable cure $[2,3]$. It has become an acceptable and effective surgical approach over the last two decades and avoids the need to perform an extensive bilateral neck dissection [2-4]. This approach is used primarily in patients who clinically and radiographically appear to have a single hyperfunctioning gland [6]. It is not generally recommended in patients with suspected multigland disease [6]. Directed parathyroidectomy has the advantages of shorter operative times, decreased surgical dissection, lower costs, shorter hospital stays and a decreased risk of transient postoperative hypocalcemia [2-3,62,63] compared with bilateral neck explorations [63]. This approach can be accomplished through a small midline or lateral cervical incision. During the procedure, it is recommended to use intraoperative adjuncts to avoid failure rates [6]. Rapid intraoperative PTH assays are commonly employed in which a serum PTH is drawn pre-skin-incision, pre-gland-excision and then 5 min post-gland-excision, 10 and 20 min post-gland-excision. The 'Miami criterion' is a validated tool that successfully predicts postoperative eucalcemia with an accuracy of $96 \%$ for over 6 months $[60,64,65]$ and is satisfied when there is a 50\% decline in the postexcision PTH values compared with either of the pre-excision values [60]. If the postexcision PTH values fail to decline by $50 \%$, further investigation and excision of the pathologic parathyroid gland is required with repeated intraoperative PTH monitoring. Directed parathyroidectomy with intraoperative PTH monitoring results in curative rates that exceed $98 \%$ with nerve injury rates of less than $1 \%[2-4]$.

Other available intraoperative adjuncts include ultrasonography, jugular venous sampling, $\gamma$-probe localization and frozen sections. Cervical ultrasound and/or PTH evaluation by jugular venous sampling can be performed by the surgeon in the operating room prior to incision, which has been shown to be particularly useful for identifying parathyroid adenomas in sestamibi-negative patients [54,66]. In these cases, the surgeon can then proceed with a 
directed parathyroidectomy and afford the patient a bilateral exploration. Intraoperative $\gamma$ probe may also aid in the detection of abnormal glands by demonstrating increased activity of a pathologic gland compared with background radiation levels during the procedure. This can be useful in cases of intrathyroidal or ectopic glands poorly visualized at the time of resection [67]. Although success rates vary, there is overall accuracy in localization with this adjunct [67-70]. Most surgeons do not regularly employ $\gamma$-probe localization due to the increased expense and need for specific personnel and institutional expertise. Prospective studies comparing intraoperative PTH monitoring to $\gamma$-probe localization demonstrate both having high sensitivities, accuracies and positive predictive values, however intraoperative PTH testing exceeds $\gamma$ probe in all categories [70]. Frozen section analysis is not generally recommended, as it does not provide any information regarding residual hyperfunctioning parathyroid tissue in situ. If the surgeon is in doubt of the tissue of origin of a specimen, frozen section can be helpful to distinguish thyroid nodule from parathyroid from lymph node or thymic tissue. However given the added time and cost, routine use of frozen section is not advised.

The University of Miami group has extensively compared long term outcome data in 718 patients who underwent either four-gland exploration $(\mathrm{n}=335)$ between 1969 and 1993 with an average follow up of 10 years or focused parathyroidectomy with intraoperative PTH monitoring $(n=383)$ between 1993 and 2003 with an average follow up of 3 years [54]. Of note, all patients with sporadic PHPT between 1993 and 2003 underwent focused parathyroidectomy even if preoperative localization studies were negative $(5 \%)$ or equivocal $(13 \%)$. For these patients, PTH levels from jugular vein sampling prior to skin incision were drawn and often pointed to the correct side harboring the abnormal gland. This allowed for a successful unilateral neck exploration. Operative failures, defined as hypercalcemia and high PTH levels within 6 months of surgery, were found in $6 \%$ of the bilateral neck exploration group (94\% success rate), compared with $3 \%$ in the focused parathyroidectomy group ( $97 \%$ success rate, $\mathrm{p}=0.04)$. Most recurrences occurred later than 30 months, reported as $4 \%$ in the bilateral neck exploration group and 3\% in the focused parathyroidectomy group. The incidence of multigland disease was higher in the bilateral neck exploration group when based on size (10\%), however similar rates were found when based on function (3\% in both groups). In the focused parathyroidectomy group, intraoperative PTH monitoring recognized 10 of 12 patients with multigland disease (83\%, two false positive results). Other studies have reported various frequencies of multigland disease discovered at the time of surgery, ranging from 5 to $33 \%$, similarly depending on the classification criteria and operative technique [71]. Although bilateral neck exploration has been shown to discover multigland disease at a higher rate, there is no statistical difference in success rates between the two approaches when reviewing multiple series $[2,59,71]$.

Intraoperative neuromonitoring for recurrent laryngeal nerve function is advocated by some surgeons but requires general anesthesia with endotracheal intubation. This is usually considered when a concomitant thyroid procedure is needed or in cases of reoperation. It is not usually indicated for routine parathyroid procedures. Transcervicalmediastinal and thorascopic approaches are often indicated for ectopic and mediastinal glands. If normal parathyroid glands appear devascularized during the procedure, immediate autotransplantation is recommended.

When parathyroid cancer is suspected, an en bloc resection without violating the parathyroid capsule is the standard of care [6], often necessitating a thyroid lobectomy when the gland is abutting the thyroid gland. There is insufficient evidence to recommend a prophylactic central neck dissection during this resection. Adjuvant radiation therapy is not routinely recommended for parathyroid cancer but is instead reserved for palliative care options [6].

\section{Operative considerations for hereditary PHPT}

When performing parathyroid surgery on patients with a known hereditary condition, bilateral exploration is usually the recommended approach, with some exceptions. The operation is often more challenging due to the nature of a more extensive surgery while balancing the goals of achieving normocalcemia and avoiding permanent hypoparathyroidism [2,6,72]. These patients are at a higher risk for recurrent disease making re-operations more difficult $[2,72]$. The surgeon must take into consideration each hereditary condition and appropriately adjust the therapeutic approach.

Patients with MEN 1 syndrome are likely to have other endocrinopathies such as pituitary, pancreaticoduodenal, thymic and bronchial carcinoid tumors that can be addressed at the time of parathyroidectomy [2]. A subtotal parathyroidectomy is the treatment of choice, excising 3.5 glands and leaving a viable remnant from the most normal appearing parathyroid with a marking clip or suture along with concurrent cervical thymectomy [72-75]. Cervical thymectomy is recommended for MEN 1 patients due to a $15 \%$ chance of discovering parathyroid tissue in the thymus and the higher rate of thymic carcinoid tumors in this population $[2,76]$. An acceptable alternative is to 
perform a total parathyroidectomy with upper extremity autotransplantation, however this is associated with higher rates of permanent hypoparathyroidism $[2,77]$. While this approach potentially avoids recurrent disease in the neck, forearm autografts are also at risk for hyperplasia which can be difficult to distinguish in the setting of simultaneous cervical recurrence [2]. Directed parathyroidectomy with intraoperative PTH monitoring can be considered only if preoperative localization studies clearly demonstrate a single enlarged gland [78]. However this places the patient at higher risk for persistent or recurrent disease [2,77]. Another described approach involves removing both glands from the ipsilateral neck along with the cervical thymus. If recurrence occurs, reoperation is focused in the contralateral neck with less scarring, however this risks removing the most normal parathyroid gland that may have been the ideal remnant [2]. Preliminary findings of the DutchMEN 1 study has correlated specific MEN 1 genotypes with the risk of persistent or recurrent disease [77] which can help guide the operative approach. For all reoperations, preoperative vocal cord evaluation and cryopreservation of resected parathyroid tissue is highly recommended [76]. Percutaneous ethanol ablation can be an effective treatment option for MEN 1 syndrome patients with recurrent hypercalcemia who have been difficult to manage surgically $[79,80]$.

In contrast to MEN 1, MEN 2A patients are less likely to experience recurrent disease [2], and a directed parathyroidectomy is recommended with concurrent total thyroidectomy for treatment or prophylaxis of medullary thyroid cancer $[22,72,75,81]$. Screening for a pheochromocytoma followed by adrenalectomy when indicated takes precedence over thyroidectomy and parathyroidectomy surgeries.

Patients with HPT-JT syndrome require detection and treatment for renal cysts, ossifying jaw fibromas, uterine carcinoma and adult Wilm's tumors in addition to PHPT and they are at a much higher risk for parathyroid carcinoma. Patients usually present with marked hypercalcemia and enlarged, often cystic parathyroid glands [2]. The risk of parathyroid cancer is $10-15 \%$ in HPT-JT [82], which typically manifests as a hard, fibrotic gland with dense adhesions to surrounding structures despite no prior history of neck surgery or radiation. When cancer is suspected, an en bloc resection including any invaded muscles with a concurrent thyroid lobectomy is the standard of care $[2,83]$. In the setting of recurrent disease, the surgeon must consider local cancer recurrence or a new adenoma, both treated with repeat resection, along with systemic metastasis, treated with calcimimetics and possible chemotherapy [2].

Patients with hereditary PHPT often go unrecognized prior to surgery, especially when no family history exists. A higher level of suspicion must be present when patients are younger (less than 45 years) with a history of failed prior parathyroidectomy operation(s) and negative preoperative localization studies. Genetic testing and biochemical screening is recommended when the suspicion is present [2].

\section{Postoperative care \& complications}

The overall perioperative morbidity rate from a parathyroidectomy is less than $4 \%$ and the mortality rate approaches $0 \%$ when performed by an experienced surgeon [2], with higher complication rates occurring in elderly patients receiving general anesthesia. Wound infections and postoperative hematomas are rare [2], however the latter can become a life threatening emergency requiring urgent surgical decompression. Recurrent laryngeal nerve injury rates are $1 \%$ in most reports [2]. If there is concern for airway comprise postoperatively, direct laryngoscopy with vocal cord evaluation is indicated. Rates of postoperative hypocalcemia range from 5 to $52 \%[2,6,84]$. The number of parathyroid glands removed is the single predictive factor for hypocalcemia [84]. Mild and severe forms of hypocalcemia are more common in patients who undergo bilateral exploration versus a directed parathyroidectomy $[6,85]$. Postoperative hypocalcemia is usually transient and mild, which can be treated in the outpatient setting. It is more common in those with vitamin D deficiency or malabsorptive conditions such as prior bariatric bypass surgery, celiac sprue, Crohn's disease or other bowel resection surgeries [6]. This can cause readmissions, prolonged hospital stays and emergency department visits. Clear patient communication, education about expectations and resources should be provided to each individual. Short term calcium and/or vitamin D supplementation should be considered for prophylaxis against postoperative hypocalcemia [6]. Patients with prolonged hypoparathyroidism may require calcium and calcitriol treatment for 6-12 months and may become eligible for recombinant PTH therapy [6]. Severe hypocalcemia is rare [6], in which the patient may experience perioral and distal extremity paresthesias, often requiring intravenous calcium replacement. In patients who are vitamin-D-deficient, it is important to provide vitamin $\mathrm{D}$ supplementation after surgery to prevent hypocalcemia due to hungry bone syndrome [6,40]. Replenishing vitamin D helps with calcium absorption, PTH normalization and may possibly improve BMD [6]. Permanent hypoparathyroidism is rare after an initial parathyroidectomy with rates of $0-3.6 \%[6,85]$. 


\section{Long term monitoring}

Operative failure is defined as persistent hypercalcemia within the 6 months immediately following surgery, with rates ranging between 3 and 6\% [6,54]. Recurrent disease is defined as hypercalcemia returning more than 6 months after parathyroidectomy when eucalcemia had initially been achieved. Average recurrence rates are $2-3 \%$ [3]. For patients with normocalcemia PHPT, PTH concentrations must decrease to normal ranges by 6 months, otherwise causes of secondary hyperparathyroidism or persistent disease must be considered. There are several causes for persistent elevated PTH levels postoperatively with normal calcium concentrations, such as vitamin D deficiency, downregulation of PTH sensors, and the presence of chronic kidney disease [86]. Long term follow-up is recommended to evaluate for complications and cure [6] with biochemical testing of serum calcium, PTH, vitamin D, phosphorus for the first 6-month period after surgery, followed by annual serum calcium levels thereafter.

In cases of persistent or recurrent disease, re-operations are often indicated [6]; however, cure rates decline to 82-98\% with a higher incidence of recurrent laryngeal nerve injuries compared with the initial operation $[6,87]$. More strict criteria, indications and preoperative evaluation takes place prior to any subsequent surgeries and referral to a high volume center is indicated. It is recommended to reconfirm the biochemical diagnosis, obtain all pertinent records including operative reports and evaluate RLN function. Most surgeons require two studies showing concordance.

\section{Conclusion \& future perspective}

The management and approach to PHPT has significantly evolved over the last two decades and continues to change as technology advances and our understanding of the disease process grows. It is clear that surgery is the only curative treatment option for both symptomatic and asymptomatic PHPT, which has been shown to improve and potentially resolve metabolic derangements and clinical symptoms $[6,10,24,31-38]$. Therefore, referral to an experienced parathyroid surgeon is warranted in every case. It is important to keep in mind that PHPT is a biochemical diagnosis, not based on imaging studies, and the surgeon is ideally the one to obtain imaging studies for the sole purpose of surgical planning. Directed parathyroidectomy with intraoperative PTH monitoring is an effective surgical approach, however bilateral exploration is still a commonly used approach under certain circumstances.

Future research is needed to develop a validated tool to interpret PTH values based on variations of vitamin D levels, age, race, sex, BMI and menopausal status. Increased education and global access to screening will continue to allow for earlier disease detection and treatment of asymptomatic patients prior to the development of clinical manifestations. Understanding the most updated guidelines, especially as it pertains to asymptomatic PHPT patients, will also improve the management and individualized approach to this disease.

\section{Executive summary}

- Primary hyperparathyroidism (PHPT) is a biochemical diagnosis consisting of an elevated serum calcium and parathyroid hormone (PTH) in the setting of normal renal function.

- When evaluating a patient for PHPT, measure: serum calcium, PTH, creatinine, 25-hydroxyvitamin D and 24-h urinary calcium and creatinine.

- Be familiar with the three common biochemical presentations of PHPT:

- Elevated calcium and PTH (classic form);

- Elevated calcium and normal PTH (nonsuppressed PTH);

- Normal calcium and elevated PTH (normocalcemic PHPT).

- Exclude familial hypocalciuric hypercalcemia and disorders causing secondary hyperparathyroidism.

- There is usually evidence of end-organ damage such as nephrolithiasis, reductions in bone mineral density or decreased renal function even in the asymptomatic patient.

- Surgery is the only curative treatment option.

- Preoperative imaging is only used for surgical planning and typically includes a cervical ultrasound and sestamibi scan or 4-dimension computed tomography.

- Negative or nonlocalizing imaging should not inhibit referral to an experienced parathyroid surgeon.

- Directed parathyroidectomy with intraoperative PTH monitoring has similar success rates as bilateral neck exploration with the advantages of shorter operative times, decreased surgical dissection, lower costs, shorter hospital stays and a decreased risk of transient postoperative hypocalcemia. 
Financial \& competing interests disclosure

The authors have no relevant affiliations or financial involvement with any organization or entity with a financial interest in or financial conflict with the subject matter or materials discussed in the manuscript. This includes employment, consultancies, honoraria, stock ownership or options, expert testimony, grants or patents received or pending, or royalties.

No writing assistance was utilized in the production of this manuscript.

\section{Open access}

This work is licensed under the Attribution-NonCommercial-NoDerivatives 4.0 Unported License. To view a copy of this license, visit http://creativecommons.org/licenses/by-nc-nd/4.0/

\section{References}

Papers of special note have been highlighted as: $\bullet$ of interest

1. Delellis RA, Mazzaglia P, Mangray S. Primary hyperparathyroidism: a current perspective. Arch. Pathol. Lab. Med. 132(8), 1251-1262 (2008).

2. Udelsman R, Akerstrom G, Biagini C et al. The surgical management of asymptomatic primary hyperparathyroidism: proceedings of the fourth international workshop. J. Clin. Endocrinol. Metab. 99(10), 3595-3606 (2014).

- A committee from the Fourth International Workshop on the Management of Asymptomatic Primary Hyperparathyroidism held in Florence, Italy, in 2013 developed key questions regarding controversial topics pertaining to the surgical management of asymptomatic primary hyperparathyroidism (PHPT). This article summarizes the consensus regarding those surgical questions and provides an in-depth discussion on the management of hereditary forms of PHPT, such as MEN 1, MEN 2A, MEN 2B, rare MEN 4 and hyperparathyroidism-jaw tumor syndromes.

3. Udelsman R, Lin Z, Donovan P. The superiority of minimally invasive parathyroidectomy based on 1650 consecutive patients with primary hyperparathyroidism. Ann. Surg. 253(3), 585-591 (2011).

4. Van Udelsman B, Udelsman R. Surgery in primary hyperparathyroidism: extensive personal experience. J. Clin. Densitom. 16(1), 54-59 (2013).

5. Bilezikian JP, Brandi ML, Eastell R et al. Guidelines for the management of asymptomatic primary hyperparathyroidism: summary statement from the Fourth International Workshop. J. Clin. Endocrinol. Metab. 99(10), 3561-3569 (2014).

- Provides revised guidelines from the Fourth International Workshop on the Management of Asymptomatic Primary Hyperparathyroidism suggesting a more extensive evaluation of renal and skeletal systems to be adopted as part of the indications for surgery. It also provides recommendations for monitoring patients who do not meet surgical criteria.

6. Wilhelm SM, Wang TS, Ruan DT et al. The American Association of Endocrine Surgeons' guidelines for definitive management of primary hyperparathyroidism. JAMA Surg. 151(10), 959-968 (2016).

- The American Association of Endocrine Surgeons published updated evidence-based guidelines for the diagnosis and management of PHPT, incorporating the most recent literature using an extensive PubMed search. The recommendations specifically address the surgical aspects of PHPT and offer guidance for every step of clinical management, from diagnosis to postoperative care.

7. Eastell R, Brandi ML, Costa AG, D’Amour P, Shoback DM, Thakker RV. Diagnosis of asymptomatic primary hyperparathyroidism: proceedings of the fourth international workshop. J. Clin. Endocrinol. Metab. 99(10), 3570-3579 (2014).

- A committee from the Fourth International Workshop on the Management of Asymptomatic Primary Hyperparathyroidism provides consensus recommendations on the specific tests used to diagnose PHPT, including PTH reference ranges, PTH assays and vitamin $\mathrm{D}$ concentrations. It also summarizes various biochemical presentations and provides guidelines on genetic testing.

8. Cusano NE, Silverberg SJ, Bilezikian JP. Normocalcemic primary hyperparathyroidism. J. Clin. Densitom. 16(1), 33-39 (2013).

9. Rejnmark L, Amstrup AK, Mollerup CL, Heickendorff L, Mosekilde L. Further insights into the pathogenesis of primary hyperparathyroidism: a nested case-control study. J. Clin. Endocrinol. Metab. 98(1), 87-96 (2013).

10. Rubin MR, Bilezikian JP, Mcmahon DJ et al. The natural history of primary hyperparathyroidism with or without parathyroid surgery after 15 years. J. Clin. Endocrinol. Metab. 93(9), 3462-3470 (2008).

11. Grubbs EG, Rafeeq S, Jimenez C et al. Preoperative vitamin D replacement therapy in primary hyperparathyroidism: safe and beneficial? Surgery 144(6), 852-858 (2008).

12. Tucci JR. Vitamin D therapy in patients with primary hyperparathyroidism and hypovitaminosis D. Eur. J. Endocrinol. 161(1), 189-193 (2009).

13. Isidro ML, Ruano B. Biochemical effects of calcifediol supplementation in mild, asymptomatic, hyperparathyroidism with concomitant vitamin D deficiency. Endocrine 36(2), 305-310 (2009).

14. Souberbielle JC, Brazier F, Piketty ML, Cormier C, Minisola S, Cavalier E. How the reference values for serum parathyroid hormone concentration are (or should be) established? J. Endocrinol. Invest. 40(3), 241-256 (2017). 
15. Holick MF, Binkley NC, Bischoff-Ferrari HA et al. Evaluation, treatment, and prevention of vitamin D deficiency: an Endocrine Society clinical practice guideline. J. Clin. Endocrinol. Metab. 96(7), 1911-1930 (2011).

16. Eastell R, Arnold A, Brandi ML et al. Diagnosis of asymptomatic primary hyperparathyroidism: proceedings of the third international workshop. J. Clin. Endocrinol. Metab. 94(2), 340-350 (2009).

17. Harvey A, Hu M, Gupta M et al. A new, vitamin D-based, multidimensional nomogram for the diagnosis of primary hyperparathyroidism. Endocr. Pract. 18(2), 124-131 (2012).

18. Jin J, Mitchell J, Shin J, Berber E, Siperstein AE, Milas M. Calculating an individual maxPTH to aid diagnosis of normocalemic primary hyperparathyroidism. Surgery 152(6), 1184-1192 (2012).

19. Fillee C, Keller T, Mourad M, Brinkmann T, Ketelslegers JM. Impact of vitamin D-related serum PTH reference values on the diagnosis of mild primary hyperparathyroidism, using bivariate calcium/PTH reference regions. Clin. Endocrinol. (Oxf.) 76(6), 785-789 (2012).

20. Amstrup AK, Rejnmark L, Vestergaard P et al. Vitamin D status, physical performance and body mass in patients surgically cured for primary hyperparathyroidism compared with healthy controls - a cross-sectional study. Clin. Endocrinol. (Oxf.) 74(1), 130-136 (2011).

21. Gunn IR, Gaffney D. Clinical and laboratory features of calcium-sensing receptor disorders: a systematic review. Ann. Clin. Biochem. 41(Pt 6), 441-458 (2004).

22. Udelsman R, Donovan P, Shaw C. Cure predictability during parathyroidectomy. World J. Surg. 38(3), 525-533 (2014).

23. Nesbit MA, Hannan FM, Howles SA et al. Mutations affecting G-protein subunit alpha11 in hypercalcemia and hypocalcemia. N. Engl. J. Med. 368(26), 2476-2486 (2013).

24. Silverberg SJ, Clarke BL, Peacock M et al. Current issues in the presentation of asymptomatic primary hyperparathyroidism: proceedings of the Fourth International Workshop. J. Clin. Endocrinol. Metab. 99(10), 3580-3594 (2014).

- Summarizes data on the various traditional and nontraditional manifestations of PHPT according to the expert panel consensus of the Fourth International Workshop on the Management of Asymptomatic Primary Hyperparathyroidism. This report provides information on the natural history of PHPT, geographic differences of predominant phenotypes and various organ system involvement of PHPT.

25. Starker LF, Akerstrom T, Long WD et al. Frequent germ-line mutations of the MEN1, CASR, and HRPT2/CDC73 genes in young patients with clinically non-familial primary hyperparathyroidism. Horm. Cancer 3(1-2), 44-51 (2012).

26. Sharma J, Weber CJ. Surgical therapy for familial hyperparathyroidism. Am. Surg. 75(7), 579-582 (2009).

27. Westin G, Bjorklund P, Akerstrom G. Molecular genetics of parathyroid disease. World J. Surg. 33(11), 2224-2233 (2009).

28. Howell VM, Haven CJ, Kahnoski K et al. HRPT2 mutations are associated with malignancy in sporadic parathyroid tumours. J. Med. Genet. 40(9), 657-663 (2003).

29. Pandya C, Uzilov AV, Bellizzi J et al. Genomic profiling reveals mutational landscape in parathyroid carcinomas. JCI Insight 2(6), e92061 (2017).

30. Costa-Guda J, Marinoni I, Molatore S, Pellegata NS, Arnold A. Somatic mutation and germline sequence abnormalities in CDKN1B, encoding p27Kip1, in sporadic parathyroid adenomas. J. Clin. Endocrinol. Metab. 96(4), E701-E706 (2011).

31. Mollerup CL, Vestergaard P, Frokjaer VG, Mosekilde L, Christiansen P, Blichert-Toft M. Risk of renal stone events in primary hyperparathyroidism before and after parathyroid surgery: controlled retrospective follow up study. BMJ 325(7368), 807 (2002).

32. Murray SE, Pathak PR, Pontes DS et al. Timing of symptom improvement after parathyroidectomy for primary hyperparathyroidism. Surgery 154(6), 1463-1469 (2013).

33. Reiher AE, Mazeh H, Schaefer S, Gould J, Chen H, Sippel RS. Symptoms of gastroesophageal reflux disease improve after parathyroidectomy. Surgery 152(6), 1232-1237 (2012).

34. Broulik PD, Broulikova A, Adamek S et al. Improvement of hypertension after parathyroidectomy of patients suffering from primary hyperparathyroidism. Int. J. Endocrinol. 2011, 309068 (2011).

35. Heyliger A, Tangpricha V, Weber C, Sharma J. Parathyroidectomy decreases systolic and diastolic blood pressure in hypertensive patients with primary hyperparathyroidism. Surgery 146(6), 1042-1047 (2009).

36. Mcmahon DJ, Carrelli A, Palmeri N et al. Effect of parathyroidectomy upon left ventricular mass in primary hyperparathyroidism: a meta-analysis. J. Clin. Endocrinol. Metab. 100(12), 4399-4407 (2015).

37. Nilsson IL, Aberg J, Rastad J, Lind L. Maintained normalization of cardiovascular dysfunction 5 years after parathyroidectomy in primary hyperparathyroidism. Surgery 137(6), 632-638 (2005).

38. Almqvist EG, Bondeson AG, Bondeson L, Nissborg A, Smedgard P, Svensson SE. Cardiac dysfunction in mild primary hyperparathyroidism assessed by radionuclide angiography and echocardiography before and after parathyroidectomy. Surgery 132(6), 1126-1132 (2002).

39. Cohen MS, Finkelstein SE, Brunt LM et al. Outpatient minimally invasive parathyroidectomy using local/regional anesthesia: a safe and effective operative approach for selected patients. Surgery 138(4), 681-687 (2005). 
40. Marcocci C, Bollerslev J, Khan AA, Shoback DM. Medical management of primary hyperparathyroidism: proceedings of the fourth International Workshop on the Management of Asymptomatic Primary Hyperparathyroidism. J. Clin. Endocrinol. Metab. 99(10), 3607-3618 (2014).

- Provides updated recommendations developed at the Fourth International Workshop on the Management of Asymptomatic Primary Hyperparathyroidism on calcium and vitamin D supplementation in patients with PHPT along with medical management for patients who do not undergo surgery.

41. Woll ML, Mazeh H, Anderson BM, Chen H, Sippel RS. Breast radiation correlates with side of parathyroid adenoma. World J. Surg. 36(3), 607-611 (2012).

42. Schneider AB, Shore-Freedman E, Weinstein RA. Radiation-induced thyroid and other head and neck tumors: occurrence of multiple tumors and analysis of risk factors. J. Clin. Endocrinol. Metab. 63(1), 107-112 (1986).

43. Woll M, Sippel RS, Chen H. Does previous head and neck irradiation increase the chance of multigland disease in patients with hyperparathyroidism? Ann. Surg. Oncol. 18(8), 2240-2244 (2011).

44. Woo JW, Kim SK, Park I, Choe JH, Kim JH, Kim JS. A novel gel pad laryngeal ultrasound for vocal cord evaluation. Thyroid 27(4), 553-557 (2017).

45. Woo JW, Park I, Choe JH, Kim JH, Kim JS. Comparison of ultrasound frequency in laryngeal ultrasound for vocal cord evaluation. Surgery 161(4), 1108-1112 (2017).

46. Awad SS, Miskulin J, Thompson N. Parathyroid adenomas versus four-gland hyperplasia as the cause of primary hyperparathyroidism in patients with prolonged lithium therapy. World J. Surg. 27(4), 486-488 (2003).

47. Starker LF, Bjorklund P, Theoharis C, Long WD 3rd, Carling T, Udelsman R. Clinical and histopathological characteristics of hyperparathyroidism-induced hypercalcemic crisis. World J. Surg. 35(2), 331-335 (2011).

48. Kunstman JW, Kirsch JD, Mahajan A, Udelsman R. Clinical review: parathyroid localization and implications for clinical management. J. Clin. Endocrinol. Metab. 98(3), 902-912 (2013).

49. Solorzano CC, Carneiro-Pla D. Minimizing cost and maximizing success in the preoperative localization strategy for primary hyperparathyroidism. Surg. Clin. North Am. 94(3), 587-605 (2014).

50. Lubitz CC, Hunter GJ, Hamberg LM et al. Accuracy of 4-dimensional computed tomography in poorly localized patients with primary hyperparathyroidism. Surgery 148(6), 1129-1137 (2010).

51. Wang TS, Cheung K, Farrokhyar F, Roman SA, Sosa JA. Would scan, but which scan? A cost-utility analysis to optimize preoperative imaging for primary hyperparathyroidism. Surgery 150(6), 1286-1294 (2011).

52. Morita SY, Somervell H, Umbricht CB, Dackiw AP, Zeiger MA. Evaluation for concomitant thyroid nodules and primary hyperparathyroidism in patients undergoing parathyroidectomy or thyroidectomy. Surgery 144(6), 862-866 (2008).

53. Summers GW. Parathyroid update: a review of 220 cases. Ear Nose Throat J. 75(7), 434-439 (1996).

54. Irvin GL III, Carneiro DM, Solorzano CC. Progress in the operative management of sporadic primary hyperparathyroidism over 34 years. Ann. Surg. 239(5), 704-708 (2004).

55. Zarebczan B, Chen H. Influence of surgical volume on operative failures for hyperparathyroidism. Adv. Surg. 45, 237-248 (2011).

56. Mitchell J, Milas M, Barbosa G, Sutton J, Berber E, Siperstein A. Avoidable reoperations for thyroid and parathyroid surgery: effect of hospital volume. Surgery 144(6), 899-906 (2008).

57. Chen H, Wang TS, Yen TW et al. Operative failures after parathyroidectomy for hyperparathyroidism: the influence of surgical volume. Ann. Surg. 252(4), 691-695 (2010).

58. Abdulla AG, Ituarte PH, Harari A, Wu JX, Yeh MW. Trends in the frequency and quality of parathyroid surgery: analysis of 17,082 cases over 10 years. Ann. Surg. 261(4), 746-750 (2015).

59. Allendorf J, Digorgi M, Spanknebel K, Inabnet W, Chabot J, Logerfo P. 1112 consecutive bilateral neck explorations for primary hyperparathyroidism. World J. Surg. 31(11), 2075-2080 (2007).

60. Irvin GL 3rd, Prudhomme DL, Deriso GT, Sfakianakis G, Chandarlapaty SK. A new approach to parathyroidectomy. Ann. Surg. 219(5), 574-579 (1994).

61. Siperstein A, Berber E, Barbosa GF et al. Predicting the success of limited exploration for primary hyperparathyroidism using ultrasound, sestamibi, and intraoperative parathyroid hormone: analysis of 1158 cases. Ann. Surg. 248(3), 420-428 (2008).

62. Burkey SH, Snyder WH III, Nwariaku F, Watumull L, Mathews D. Directed parathyroidectomy: feasibility and performance in 100 consecutive patients with primary hyperparathyroidism. Arch. Surg. 138(6), 604-608 (2003).

63. Schneider DF, Mazeh H, Sippel RS, Chen H. Is minimally invasive parathyroidectomy associated with greater recurrence compared with bilateral exploration? Analysis of more than 1,000 cases. Surgery 152(6), 1008-1015 (2012).

64. Johnson LR, Doherty G, Lairmore T et al. Evaluation of the performance and clinical impact of a rapid intraoperative parathyroid hormone assay in conjunction with preoperative imaging and concise parathyroidectomy. Clin. Chem. 47(5), 919-925 (2001).

65. Carneiro DM, Solorzano CC, Irvin GL III. Recurrent disease after limited parathyroidectomy for sporadic primary hyperparathyroidism. J. Am. Coll. Surg. 199(6), 849-853 (2004). 
66. Livingston CD, Victor B, Askew R et al. Surgeon-performed ultrasonography as an adjunct to minimally invasive radio-guided parathyroidectomy in 100 consecutive patients with primary hyperparathyroidism. Endocr. Pract. 14(1), 28-32 (2008).

67. Mcveigh T, Lowery AJ, Quill DS, Kerin MJ. Changing practices in the surgical management of hyperparathyroidism - a 10-year review. Surgeon 10(6), 314-320 (2012).

68. Rubello D, Mariani G, Pelizzo MR. Minimally invasive radio-guided parathyroidectomy on a group of 452 primary hyperparathyroid patients: refinement of preoperative imaging and intraoperative procedure. Nuklearmedizin 46(3), 85-92 (2007).

69. Burkey SH, Van Heerden JA, Farley DR, Thompson GB, Grant CS, Curlee KJ. Will directed parathyroidectomy utilizing the gamma probe or intraoperative parathyroid hormone assay replace bilateral cervical exploration as the preferred operation for primary hyperparathyroidism? World J. Surg. 26(8), 914-920 (2002).

70. Chen H, Mack E, Starling JR. A comprehensive evaluation of perioperative adjuncts during minimally invasive parathyroidectomy: which is most reliable? Ann. Surg. 242(3), 375-380 (2005).

71. McGill J, Sturgeon C, Kaplan SP, Chiu B, Kaplan EL, Angelos P. How does the operative strategy for primary hyperparathyroidism impact the findings and cure rate? A comparison of 800 parathyroidectomies. J. Am. Coll. Surg. 207(2), 246-249 (2008).

72. Carling T, Udelsman R. Parathyroid surgery in familial hyperparathyroid disorders. J. Intern. Med. 257(1), 27-37 (2005).

73. Stalberg P, Carling T. Familial parathyroid tumors: diagnosis and management. World J. Surg. 33(11), 2234-2243 (2009).

74. Schreinemakers JM, Pieterman CR, Scholten A, Vriens MR, Valk GD, Rinkes IH. The optimal surgical treatment for primary hyperparathyroidism in MEN1 patients: a systematic review. World J. Surg. 35(9), 1993-2005 (2011).

75. Twigt BA, Scholten A, Valk GD, Rinkes IH, Vriens MR. Differences between sporadic and MEN related primary hyperparathyroidism; clinical expression, preoperative workup, operative strategy and follow-up. Orphanet J. Rare Dis. 8, 50 (2013).

76. Vanderwalde LH, Haigh PI. Surgical approach to the patient with familial hyperparathyroidism. Curr. Treat. Options Oncol. 7(4), 326-333 (2006).

77. Pieterman CR, Van Hulsteijn LT, Den Heijer M et al. Primary hyperparathyroidism in MEN1 patients: a cohort study with longterm follow-up on preferred surgical procedure and the relation with genotype. Ann. Surg. 255(6), 1171-1178 (2012).

78. Kraimps JL, Duh QY, Demeure M, Clark OH. Hyperparathyroidism in multiple endocrine neoplasia syndrome. Surgery 112(6), 1080-1086 (1992).

79. Singh Ospina N, Thompson GB, Lee RA, Reading CC, Young WF, Jr. Safety and efficacy of percutaneous parathyroid ethanol ablation in patients with recurrent primary hyperparathyroidism and multiple endocrine neoplasia Type 1. J. Clin. Endocrinol. Metab. 100(1), E87-E90 (2015).

80. Chen HH, Lin CJ, Wu CJ et al. Chemical ablation of recurrent and persistent secondary hyperparathyroidism after subtotal parathyroidectomy. Ann. Surg. 253(4), 786-790 (2011).

81. Scholten A, Schreinemakers JM, Pieterman CR, Valk GD, Vriens MR, Borel Rinkes IH. Evolution of surgical treatment of primary hyperparathyroidism in patients with multiple endocrine neoplasia type 2A. Endocr. Pract. 17(1), 7-15 (2011).

82. Gimm O, Lorenz K, Nguyen Thanh P et al. Prophylactic parathyroidectomy for familial parathyroid carcinoma. Chirurg 77(1), 15-24 (2006).

83. Harari A, Waring A, Fernandez-Ranvier G et al. Parathyroid carcinoma: a 43-year outcome and survival analysis. J. Clin. Endocrinol. Metab. 96(12), 3679-3686 (2011).

84. Mittendorf EA, Merlino JI, McHenry CR. Post-parathyroidectomy hypocalcemia: incidence, risk factors, and management. Am. Surg. 70(2), 114-119 (2004).

85. Bergenfelz A, Lindblom P, Tibblin S, Westerdahl J. Unilateral versus bilateral neck exploration for primary hyperparathyroidism: a prospective randomized controlled trial. Ann. Surg. 236(5), 543-551 (2002).

86. Rajaei MH, Bentz AM, Schneider DF, Sippel RS, Chen H, Oltmann SC. Justified follow-up: a final intraoperative parathyroid hormone (ioPTH) Over $40 \mathrm{pg} / \mathrm{ml}$ is associated with an increased risk of persistence and recurrence in primary hyperparathyroidism. Ann. Surg. Oncol. 22(2), 454-459 (2015).

87. Karakas E, Muller HH, Schlosshauer T, Rothmund M, Bartsch DK. Reoperations for primary hyperparathyroidism - improvement of outcome over two decades. Langenbecks Arch. Surg. 398(1), 99-106 (2013). 